\title{
On the Terrestrial Propagation of ELF and VLF Waves in the Presence of a Radial Magnetic Field
}

\author{
Janis Galejs
}

\author{
Applied Research Laboratory, Sylvania Electronics System, a Division of Sylvania Electric \\ Products, Inc., 40 Sylvan Road, Waltham, Mass., 02154
}

(Received November 10, 1964)

\begin{abstract}
The continuously changing ionosphere profile is approximated by a series of homogeneous layers, and the radial field variation in the individual layers is obtained by solving a fourth order equation. Its biquadratic solutions are adequate only in regions where TE and TM modes are coupled, but more accurate solutions are required in lower layers of the ionosphere. The field components below the ionosphere are computed after multiplying a sequence of $4 \times 4$ matrices, each of which represents the effects of one of the ionosphere layers. However, simpler multiplications can be used for heights above the $D$ layer. After computing the impedance matrix at the lower boundary of the ionosphere, the propagation parameters are computed with neglected coupling between TE and TM waves below the ionosphere.

The radial magnetic field has the most pronounced effects at nighttime in the lower end of ELF range, where it increases the attenuation significantly, and also in the VLF range, where it decreases the attenuation. At daytime the presence of the radial magnetic field has only minor effects on wave propagation.
\end{abstract}

\section{Introduction}

The ionospheric anisotropy has been investigated for a model of a homogeneous ionosphere by Schumann [1955] and Wait [1962, 1963a]. The radial magnetic field causes a coupling between the TE and TM modes of propagation, but this coupling has a small effect for frequencies in the ELF and VLF range. Volland [1962, 1964] considers propagation below an inhomogeneous and anisotropic ionosphere using matrices of reflection coefficients. A formalism for treating a multilayer ionosphere has been presented by Wàit [1963b], and the formal solution for an $M$ layer ionosphere is shown to require the solution of $4 M+2$ simultaneous equations in $4 M+2$ unknowns. Another solution intended for frequencies in the Schumann resonance range $(30 \mathrm{c} / \mathrm{s}$ and lower) has been derived in the thesis of Thompson [1963], and shown by Galejs [1964a]. His procedure considers each resonant mode separately and requires the multiplication of a sequence of $4 \times 4$ matrices. This solution does not consider the displacement currents of the lower ionosphere regions and results in $Q$-factors of the Schumann resonances that are larger than observed.

In the present paper the effects of a radial magnetic field are considered with a multilayer ionosphere model. Matrix multiplication techniques are used to relate the field components at the lower ionosphere boundary to the wave amplitudes of the upper ionosphere layer. The propagation parameters of the waves in the VLF and ELF frequency bands are obtained after computing the surface impedance at the ionospheric boundary (allowing for displacement currents) and by using the appropriate modal equation in an iteration procedure. It is shown that the biquadratic solution of two coupled equations or of a single quartic equation [Wait, 1963b; Thompson, 1963; Galejs, 1964a] is not sufficient for determining the propagation parameters of the elementary waves in the lower ionosphere regions, where the TE and TM waves tend to become uncoupled by the local medium. These solutions are described in section 2 . In applying the standard technique of matrix multiplication and inversion to the upper layers of the ionosphere model, excessive cumulative errors occur particularly for frequencies in the VLF range. However, for these parameters the matrix multiplication and inversion can be replaced by a simple one term multiplication of the matrix elements, which in addition to speeding up the computational processes avoids the above convergence problem, as indicated in section 3. Numerical examples of propagation parameters for the ELF and VLF bands are shown in section 4 . 


\section{Fields in the Anisotropic Ionosphere}

The wave solutions for the fields in the anisotropic ionosphere can be derived from Maxwell's equations:

and

$$
\nabla \times \mathbf{E}=i \omega \mu_{0} \mathbf{H}
$$

$$
\nabla \times \mathbf{H}=-i \omega \epsilon_{0}[\epsilon] \mathbf{E}
$$

For an assumed $\exp (-i \omega t)$ time dependence of the fields and an $r$-directed static magnetic field the permittivity tensor $[\epsilon]$ is given by the classical magneto-ionic theory as

$$
[\epsilon]=\left[\begin{array}{ccc}
\epsilon_{3} & 0 & 0 \\
0 & \epsilon_{1} & \epsilon_{2} \\
0 & -\epsilon_{2} & \epsilon_{1}
\end{array}\right]=\left[\begin{array}{ccc}
1+\frac{i \sigma_{0}}{\omega \epsilon_{0}} & 0 & 0 \\
0 & 1+\frac{i \sigma_{1}}{\omega \epsilon_{0}} & -\frac{i \sigma_{2}}{\omega \epsilon_{0}} \\
0 & \frac{i \sigma_{2}}{\omega \epsilon_{0}} & 1+\frac{i \sigma_{1}}{\omega \epsilon_{0}}
\end{array}\right] .
$$

The conductivity components $\sigma_{0}, \sigma_{1}$, and $\sigma_{2}$ of a typical lower ionosphere model have been reported by Galejs and Row [1964] and Galejs [1964b]. The field components $E_{r}$ and $H_{r}$ are proportional to $f_{r}(\theta)=P_{\nu}(-\cos \theta)$, while $E_{\theta}, H_{\theta}, E_{\phi}$, and $H_{\phi}$ are proportional to $f_{\theta}(\theta)=f_{\phi}(\theta)=d P_{\nu}(-\cos ) / d \theta$. After writing out the 2 vector equations (1) and (2) in their 6 scalar components, the $\theta$ functions can be canceled leaving 6 scalar equations in the $r$-dependent components $E_{j}^{0}(r)=E_{j} / f_{j}(\theta), H_{j}^{0}(r)=H_{j} / f_{j}(\theta)$, where $j=r, \theta$, or $\phi$. This gives

$$
\begin{gathered}
-\nu(\nu+1) \frac{E_{\phi}^{0}}{\mathrm{r}}=i \omega \mu_{0} H_{r}^{0} \\
-\frac{1}{r} \frac{\partial}{\partial r}\left(r E_{\phi}^{0}\right)=i \omega \mu_{0} H_{\theta}^{0} \\
\frac{1}{r} \frac{\partial}{\partial r}\left(r E_{\theta}^{0}\right)-\frac{E_{r}^{0}}{r}=i \omega \mu_{0} H_{\phi}^{0} \\
\nu(\nu+1) \frac{H_{\phi}^{0}}{r}=i \omega \epsilon_{0} \epsilon_{3} E_{r}^{0} \\
\frac{1}{r} \frac{\partial}{\partial r}\left(r H_{\phi}^{0}\right)=i \omega \epsilon_{0}\left[\epsilon_{1} E_{\theta}^{0}+\epsilon_{2} E_{\phi}^{0}\right] \\
\frac{1}{r} \frac{\partial}{\partial r}\left(r H_{\theta}^{0}\right)-\frac{H_{r}^{0}}{r}=i \omega \epsilon_{0}\left[\epsilon_{2} E_{\theta}^{0}-\epsilon_{1} E_{\phi}^{0}\right] .
\end{gathered}
$$

After introducing the notation

$$
k_{j}^{2}=\omega^{2} \mu_{0} \epsilon_{0} \epsilon_{j}
$$

$(j=1,2,3)$, a manipulation of (4) to (9) results in

$$
\begin{gathered}
\frac{\partial^{3}}{\partial r^{3}}\left(r E_{\phi}^{0}\right)+k_{1}^{2} \frac{\partial}{\partial r}\left\{\left[1-\frac{\nu(\nu+1)}{k_{1}^{2} r^{2}}\right] r E_{\phi}^{0}\right\}+\frac{k_{2}^{2} k_{3}^{2}}{\nu(\nu+1)}\left[1-\frac{\nu(\nu+1)}{k_{3}^{2} r^{2}}\right] r^{2} E_{r}^{0}=0 \\
k_{2}^{2} \frac{\partial}{\partial r}\left(r E_{\phi}^{0}\right)=\frac{k_{3}^{2}}{\nu(\nu+1)}\left\{\frac{\partial^{2}}{\partial r^{2}}\left(r^{2} E_{r}^{0}\right)+k_{1}^{2}\left[1-\frac{\nu(\nu+1)}{k_{3}^{2} r^{2}}\right] r^{2} E_{r}^{0}\right\}
\end{gathered}
$$


and

$$
\left\{k_{2}^{4}+k_{1}^{4}\left[1-\frac{\nu(\nu+1)}{k_{1}^{2} r^{2}}\right]\right\} r E_{\phi}^{0}+k_{1}^{2} \frac{\partial^{2}}{\partial r^{2}}\left(r E_{\phi}^{0}\right)=k_{2}^{2} \frac{k_{3}^{2}}{\nu(\nu+1)} \frac{\partial}{\partial r}\left(r^{2} E_{r}^{0}\right) ;
$$

$E_{\phi}^{0}$ can be eliminated from (11) to (13), which gives a fourth order differential equation for computing $E_{r}^{0}$. The parameter $\nu$ is defined by $\nu(\nu+1)=\left(k_{0} a S\right)^{2}$, where $k_{0}=\omega \sqrt{\mu_{0} \epsilon_{0}}, a$ is the radius of earth, and $S$ is the yet undetermined root of the modal equation. The factors $\nu(\nu+1) /\left(k_{j} r\right)^{2}$ change gradually relative to $\left(r^{2} E_{r}^{0}\right)$, and only the first order change is considered. These factors and their first derivatives are replaced by constants

$$
\begin{gathered}
q_{j}=\frac{\nu(\nu+1)}{\left(k_{j} r_{m}\right)^{2}}, \\
q_{j}^{\prime}=\frac{\partial}{\partial r} q_{j}=-\frac{2}{r_{m}} q_{j}
\end{gathered}
$$

where $r_{m}$ is the mean radius of an ionospheric layer. This results in

$$
\frac{\partial^{4}}{\partial r^{4}}\left(r^{2} E_{r}^{0}\right)+b_{3} \frac{\partial^{3}}{\partial r^{3}}\left(r^{2} E_{r}^{0}\right)+B_{2} \frac{\partial^{2}}{\partial r^{2}}\left(r^{2} E_{r}^{0}\right)+b_{1} \frac{\partial}{\partial r}\left(r^{2} E_{r}^{0}\right)+\left(B_{0}+b_{0}\right) r^{2} E_{r}^{0}=0
$$

where

$$
\begin{aligned}
& b_{3}=\frac{q_{1}^{\prime} k_{1}^{4}}{k_{1}^{4}\left(1-q_{1}\right)+k_{2}^{4}} \\
& B_{2}=k_{1}^{2}\left(2-q_{1}-q_{3}\right) \\
& b_{1}=k_{1}^{2}\left[2 q_{3}^{\prime}+q_{1}^{\prime} \frac{k_{2}^{4}-k_{1}^{4}\left(1-q_{3}\right)}{k_{2}^{4}+k_{1}^{4}\left(1-q_{1}\right)}\right] \\
& B_{0}=k_{1}^{4}\left(1-q_{1}\right)\left(1-q_{3}\right)+k_{2}^{4}\left(1-q_{3}\right) \\
& b_{0}=-\frac{q_{1}^{\prime} q_{3}^{\prime} k_{1}^{6}}{k_{2}^{4}+k_{1}^{4}\left(1-q_{1}\right)} .
\end{aligned}
$$

A first order solution of (16) is obtained by neglecting the small terms proportional to $b_{n}$. This solution is of the form

$$
r^{2} E_{r}^{0}=e^{i k r}
$$

where

$$
k^{2}=k_{ \pm}^{2}=k_{1}^{2}\left[1-\frac{1}{2} q_{1}-\frac{1}{2} q_{3}\right] \pm i k_{2}^{2} \sqrt{1-q_{3}-\frac{1}{4}\left(\frac{k_{1}}{k_{2}}\right)^{4}\left(q_{3}-q_{1}\right)^{2}} .
$$

Equation (23) is seen to be in agreement with (34) to (36) of Wait [1963]. For negligible displacement currents $k_{j}>>k_{0}=\omega \sqrt{\mu_{0} \epsilon_{0}}$. Noting that $\nu(\nu+1) \approx\left(k_{0} r\right)^{2}, q_{j}<<1$ and (23) simplifies further to the form given by Thompson [1963].

The second order solution (designated by primes) is obtained by a single iteration substituting (22) in (16), solving for $k^{2}$, and by using (23) for $k$ in the right-hand side of this equation. This procedure gives

$$
k_{j}^{\prime 2}=\frac{1}{B_{2}}\left(k_{j}^{4}-i b_{3} k_{j}^{3}+i b_{1} k_{j}+B_{0}+b_{0}\right)=k_{j}^{2}+\frac{i k_{j}}{B_{2}}\left(b_{1}-b_{3} k_{j}^{2}\right)+\frac{b_{0}}{B_{2}}
$$


where $j=+$ or - . The second solution which corresponds to the negative of $k_{j}$ is designated as $k_{j}^{\prime \prime 2}$. Although there are only minor differences between the solutions (23) and (24), it is essential to use (24) in the subsequent expressions (35) and (48), because (23) does not result in decoupled TE and TM field components as $\epsilon_{2} \rightarrow 0$ and $\epsilon_{1}$ and $\epsilon_{3} \rightarrow 1$ in (3).

In the limiting case of $k_{2} \rightarrow 0$, (12) simplifies to

$$
\frac{\partial^{2}}{\partial r^{2}}\left(r^{2} E_{r}^{0}\right)+k_{1}^{2}\left[1-\frac{\nu(\nu+1)}{\left(k_{3} r\right)^{2}}\right]\left(r^{2} E_{r}^{2}\right)=0
$$

and, after applying (4), (11) gives

$$
\frac{\partial^{2}}{\partial r^{2}}\left(r^{2} H_{r}^{0}\right)+k_{1}^{2}\left[1-\frac{\nu(\nu+1)}{\left(k_{1} r\right)^{2}}\right]\left(r^{2} H_{r}^{0}\right)=0 .
$$

In the planar approximation (25) and (26) have solutions

$$
r^{2} E_{r}^{0}=e^{i k r}
$$

with

$$
k^{2}=k_{1}^{2}\left(1-q_{3}\right)
$$

and

$$
r^{2} H_{r}^{0}=e^{i k r}
$$

with

$$
k^{2}=k_{1}^{2}\left(1-q_{1}\right)
$$

The same solutions, (28) and (30), are also obtained for $k_{2} \rightarrow 0$ from (23). The upper and lower signs of (23) apply therefore to TM and TE field components derived from $E_{r}^{0}$ and $H_{r}^{0}$, respectively, which become coupled for $k_{2} \neq 0$.

The field components will be derived using the planar approximation, (14) and (15). The field components will be related to the amplitudes of the radial electric and magnetic field components.

The component $E_{r}$ is assumed to be of the form

$$
E_{r}^{0}=a_{\nu} \nu(\nu+1) \frac{1}{r^{2}} e^{i k r},
$$

where $k$ has four possible values according to (23). $\quad H_{\phi}^{0}$ follows from (7) as

$$
H_{\phi}^{0}=a_{\nu} i \omega \epsilon_{0} \epsilon_{3} \frac{1}{r} e^{i k r},
$$

$E_{\theta}^{0}$ is obtained from (6)

$$
E_{\theta}^{0}=\frac{a_{\nu} i\left(1-q_{3}\right) k_{3}^{2}}{k r} e^{i k r}
$$

Computing the second derivative of $\left(r H_{\theta}^{0}\right)$ from (9), applying (4) and substituting (5) and (6) for the derivatives of $\left(r E_{\phi}^{0}\right)$ and $\left(r E_{\theta}^{0}\right)$ gives

$$
H_{\theta}^{0}=a_{\nu} i \omega \epsilon_{0} \epsilon_{3} \frac{F}{r} e^{i k r}
$$


with

$$
F=\frac{k_{2}^{2}\left(1-q_{3}\right)}{\left[k^{2}-k_{1}^{2}\left(1-q_{1}\right)\right]}
$$

$E_{\phi}^{0}$ and $H_{r}^{0}$ follow from (5) and (4), respectively as

$$
\begin{aligned}
& E_{\phi}^{0}=-\frac{a_{\nu} i k_{3}^{2} F}{k r} e^{i k r} \\
& H_{r}^{0}=\frac{a_{\nu} \nu(\nu+1) k_{3}^{2} F}{\omega \mu_{0} k r^{2}} e^{i k r} .
\end{aligned}
$$

When using (24) for $k^{2}$ in (35), there is no coupling between the TM components $\left(E_{r}^{0}, E_{\theta}^{0}, H_{\phi}^{0}\right)$ and the TE components $\left(E_{\phi}^{0}, H_{r}^{0}, H_{\theta}^{0}\right)$ for $\epsilon_{2}$ or $k_{2}^{2} \rightarrow 0$ in (4) to (9), and $E_{\phi}^{0}, H_{r}^{0}$ and $H_{\theta}^{0}$ of (36), (37), and (34) approach zero. ${ }^{1}$ For negligible displacement currents, $q_{j}<<1$ and the expressions (31) to (37) are simplified to

$$
\begin{aligned}
& E_{r}^{0}=a_{\nu} \nu(\nu+1) \frac{1}{r^{2}} e^{i k r} \\
& E_{\theta}^{0}=a_{\nu} \frac{\epsilon_{3}}{\epsilon_{1} \pm i \epsilon_{2}} \frac{i k}{r} e^{i k r} \\
& E_{\phi}^{0}=a_{\nu} \frac{\epsilon_{3}}{\epsilon_{2} \pm i \epsilon_{1}} \frac{i k}{r} e^{i k r} \\
& H_{r}^{0}=-a_{\nu} \nu(\nu+1) \frac{\epsilon_{3}}{\epsilon_{2} \mp i \epsilon_{1}} \frac{k}{\omega \mu_{0} r^{2}} e^{i k r} \\
& H_{\theta}^{0}= \pm a_{\nu} \omega \epsilon_{0} \epsilon_{3} \frac{1}{r} e^{i k r} \\
& H_{\phi}^{0}=+i a_{\nu} \omega \epsilon_{0} \epsilon_{3} \frac{1}{r} e^{i k r} .
\end{aligned}
$$

These expressions of circularly polarized field components have been obtained also by Thompson [1963].

In an alternate representation, $H_{r}$ is assumed to be of the form

$$
H_{r}^{0}=b_{\nu} \nu(\nu+1) \frac{1}{r^{2}} e^{i k r}
$$

$E_{\phi}^{0}$ and $H_{\theta}^{0}$ follow from (4) and (5) as

$$
\begin{gathered}
E_{\phi}^{0}=-b_{\nu} \frac{i \omega \mu_{0}}{r} e^{i k r} \\
H_{\theta}^{0}=b_{\nu} \frac{i k}{r} e^{i k r} .
\end{gathered}
$$

\footnotetext{
${ }^{1}$ For decoupled field components, $F$ of (35) should approach zero. However, the field components are not decoupled when using (23) for $k^{2}$ in (35). For $k_{3}=k_{1}$, $F$ is finite and independent of $k_{2}$. For $k_{3} \neq k_{1}$ and $k_{2} \rightarrow 0, F \sim k_{2}^{2} /\left(k_{3}^{2}-k_{1}^{2}\right)$. After relating $k_{j}^{2}$ to the tensor conductivity components $\sigma_{j}$ by (10) and (3), $k_{j}^{2}$ can be ex pressed in terms of collision frequencies $\nu$, plasma frequencies $\omega_{0}$ and gyrofrequencies $\omega_{H}$ of the charged particles of the ionosphere, as shown by (3) to (7) of Galejs and Row [1964]. The last expression given above for $F$ becomes $F \sim \nu /\left|\omega_{H}\right|$ which is increasing with a decreasing altitude contrary to expectations.
} 
Computing the second derivative of $\left(r H_{\phi}^{0}\right)$ from (8) and applying (6), (7), and (38) gives

$$
\mathrm{E}_{r}^{0}=-\frac{b_{\nu} \nu(\nu+1) \omega \mu_{0} k E}{k_{3}^{2} r^{2}} e^{i k r}
$$

where

$$
E=\frac{k_{2}^{2}}{k^{2}-k_{1}^{2}\left(1-q_{3}\right)}
$$

$H_{\phi}^{0}$ and $E_{\theta}^{0}$ follow from (7) and (6) as

$$
\begin{gathered}
H_{\phi}^{0}=-\frac{b_{\nu} i k E}{r} e^{i k r} \\
E_{\theta}^{0}=-\frac{b_{\nu} i \omega \mu_{0} E\left(1-q_{3}\right)}{r} e^{i k r} .
\end{gathered}
$$

When using (24) for $k^{2}$ in (48), there is no coupling between the TE and TM field components if $\epsilon_{2}$ or $k_{2}^{2} \rightarrow 0$, and $E_{r}^{0}, E_{\theta}^{0}$, and $H_{\phi}^{0}$ approach zero. For negligible displacement currents, $q_{j} \ll 1$ and the expressions (47) to (50) can be simplified to

$$
\begin{aligned}
& E_{r}^{0}= \pm \frac{b_{\nu} \nu(\nu+1) i \omega \mu_{0} k}{k_{3}^{2} r^{2}} e^{i k r} \\
& E_{\theta}^{0}=\mp \frac{b_{\nu} \omega \mu_{0}}{r} e^{i k r} \\
& H_{\phi}^{0}=\mp \frac{b_{\nu} k}{r} e^{i k r} .
\end{aligned}
$$

By noting that

$$
b_{\nu}= \pm a_{\nu} k_{3}^{2} /\left(i \omega \mu_{0} k\right)
$$

the field components (38) to (43) are seen to be identical to (44) to (46) and (51) to (54).

\section{Surface Impedance of the Stratified Ionosphere}

The ionosphere is subdivided in a number of concentric spherical shells as is indicated in figure 1. The fields within the $n$th layer and on its boundaries can be represented in matrix form as

$$
\left[S_{n}(r)\right]=\left[a_{n}(r)\right] \cdot\left[C_{n}\right]
$$

where $\left[S_{n}(r)\right]$ is a column matrix of the tangential field components, $\left[C_{n}\right]$ is a column matrix of coefficients or wave amplitudes and $\left[a_{n}(r)\right]$ is a matrix of the functions or the solution matrix. At the two boundaries, $r_{n-1}$ and $r_{n}$ of the $n$th layer (55) may be written as

$$
\begin{gathered}
{\left[S_{n}\left(r_{n-1}\right)\right]=\left[a_{n}\left(r_{n-1}\right)\right] \cdot\left[C_{n}\right]} \\
{\left[S_{n}\left(r_{n}\right)\right]=\left[a_{n}\left(r_{n}\right)\right] \cdot\left[C_{n}\right] ;}
\end{gathered}
$$


FigURE 1. A spherically stratified medium.
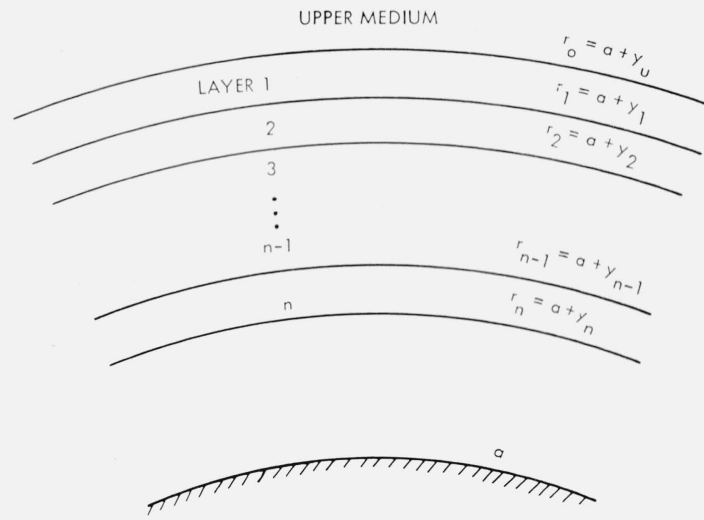

$\left[S_{n}\left(r_{n-1}\right)\right]$ is related to $\left[S_{n}\left(r_{n}\right)\right]$ by eliminating $\left[C_{n}\right]$ from (56) and (57) as

$$
\left[S_{n}\left(r_{n}\right)\right]=\left[a_{n}\left(r_{n}\right)\right] \cdot\left[a_{n}^{-1}\left(r_{n-1}\right)\right] \cdot\left[S_{n}\left(r_{n-1}\right)\right]
$$

or

$$
\left[S_{n}\left(r_{n}\right)\right]=\left[d_{n}\right] \cdot\left[S_{n}\left(r_{n-1}\right)\right]
$$

The boundary condition at $r_{n-1}$ between the layers $(n)$ and $(n-1)$ is given as

$$
\left[S_{n}\left(r_{n-1}\right)\right]=\left[S_{n-1}\left(r_{n-1}\right)\right]
$$

if there are no sources on the boundary. When combining (59) and (60), the fields at the boundary $r_{n}$ are related to the fields at the boundary $r_{0}$ as

$$
\left[S_{n}\left(r_{n}\right)\right]=\left[d_{n}\right] \cdot\left[d_{n-1}\right] \ldots\left[d_{2}\right] \cdot\left[d_{1}\right] \cdot\left[a_{u}\left(r_{0}\right)\right] \cdot\left[C_{u}\right]=\left[b_{n}\right] \cdot\left[C_{u}\right]
$$

The column matrix is assumed of the form

$$
\left[S_{n}(r)\right]=\left[\begin{array}{c}
E_{\theta}^{0}(r) \\
H_{\phi}^{0}(r) \\
E_{\phi}^{0}(r) \\
H_{\theta}^{0}(r)
\end{array}\right] .
$$

There are only upgoing waves in the upper medium and the coefficient matrix $\left[C_{u}\right]$ becomes

$$
\left[C_{u}\right]=\left[\begin{array}{c}
A \\
0 \\
B \\
0
\end{array}\right] .
$$


The matrix $\left[a_{n}(r)\right]$ is obtained from (31) to $(37)$ and (44) to (50) as

$$
\left[a_{n}(r)\right]=\left[\begin{array}{cccc}
x \exp \left(i k_{+} r\right) & -x \exp \left(-i k_{+} r\right) & -W G^{\prime} \exp \left(i k_{-} r\right) & -w G^{\prime \prime} \exp \left(-i k_{-} r\right) \\
y \exp \left(i k_{+} r\right) & y \exp \left(-i k_{+} r\right) & -z E^{\prime} \exp \left(i k_{-} r\right) & z E^{\prime \prime} \exp \left(-i k_{-} r\right) \\
-x H^{\prime} \exp \left(i k_{+}\right) & x H^{\prime \prime} \exp \left(-i k_{+} r\right) & -w \exp \left(i k_{-} r\right) & -w \exp \left(-i k_{-} r\right) \\
y F^{\prime} \exp \left(i k_{+} r\right) & y F^{\prime \prime} \exp \left(-i k_{+} r\right) & z \exp \left(i k_{-} r\right) & -z \exp \left(-i k_{-} r\right)
\end{array}\right],
$$

where

$$
\begin{aligned}
x & =\frac{i k_{3}^{2}\left(1-q_{3}\right)}{k_{+} r} \\
y & =\frac{i k_{3}^{2}}{\omega \mu_{0} r} \\
w & =\frac{i \omega \mu_{0}}{r} \\
z & =\frac{i k_{-}}{r} \\
H^{m} & =\frac{k_{2}^{2}}{\left(k_{+}^{m}\right)^{2}-k_{1}^{2}\left(1-q_{1}\right)} \\
F^{m} & =H^{m}\left(1-q_{3}\right) \\
E^{m} & =\frac{k_{2}^{2}}{\left(k_{-}^{m}\right)^{2}-k_{1}^{2}\left(1-q_{3}\right)} \\
G^{m} & =E^{m}\left(1-q_{3}\right)
\end{aligned}
$$

and where $m={ }^{\prime}$ or ". The symbols $k_{j}$ and $k_{j}^{m}$ (with $j=+$ or - ) designate solutions of (23) and (24) respectively. After computing the inverse matrix $\left[a_{n}^{-1}(r)\right]$, the matrix $\left[d_{n}\right]$ can be computed explicitly. The expressions of $\left[d_{n}\right]$ are relatively simple if $N^{\prime}=N^{\prime \prime}=N(N=E, F, G, M)$, as can be seen from appendix 1 . In the limiting cases of negligible displacement currents $\left(q_{j}<<1\right)$ and of uncoupled TE and TM modes $(N=0)$, the matrix elements are in agreement with available results [Thompson, 1963]. In the ionosphere layers where TE and TM modes are coupled the differences between $N^{\prime}$ and $N^{\prime \prime}$ can be ignored and the $\left[b_{n}\right]$ matrix of $(61)$ can be computed either by multiplying out a sequence of $\left[a_{n}\left(r_{n}\right)\right]$ and $\left[a_{n}^{-1}\left(r_{n-1}\right)\right]$ matrices or by using the explicit expressions for the $\left[d_{n}\right]$ matrix.

Thus

$$
\left[b_{n}\right]=\left[a_{n}\left(r_{n}\right)\right] \cdot\left[a_{n}^{-1}\left(r_{n-1}\right)\right] \cdot\left[b_{n-1}\right]
$$

or

$$
\left[b_{n}\right]=\left[d_{n}\right] \cdot\left[b_{n-1}\right] .
$$

In either case small elements of $\left[b_{n}\right]$ are computed by substraction of large numbers, which decreases the accuracy of $\left[b_{n}\right]$ after repeated multiplications in particular for frequencies in the VLF 
range at higher altitudes where the ionospheric wavelength becomes small. This difficulty can be avoided by evaluating the product (74) analytically, and it is shown in appendix 2 that an element $\left.b_{i j}\right|_{n}$ of $\left[b_{n}\right]$ is obtained from the corresponding element of $\left[b_{n-1}\right]$ by a single multiplication as

$$
\left.b_{i j}\right|_{n}=\left.e_{i j} \cdot b_{i j}\right|_{n-1}
$$

provided that the differences of $N(N=E, F, G, H)$ between the two adjacent layers are negligible.

Starting out with $\left[a_{n}\left(r_{0}\right)\right],\left[b_{n}\right]$ is computed initially by (75). At lower heights the computations are changed to $(73)$, and finally one obtains the matrix $\left[b_{n}\right]$ at the lower ionosphere boundary $y_{N}$ which relates the tangential field components $E_{\theta}, H_{\phi}, E_{\phi}$, and $H_{\theta}$ at this height to the amplitudes of the upgoing waves $\mathrm{A}$ and $\mathrm{B}$ of the upper ionospheric layer. The elements of an impedance matrix which characterizes the ionospheric boundary can be deduced from (61), (62), and (63) as

$$
\begin{aligned}
& Z_{11}=\left.\frac{E_{\theta}}{H_{\theta}}\right|_{H_{\phi}=0}=\frac{b_{13} b_{21}-b_{11} b_{23}}{b_{21} b_{43}-b_{23} b_{43}}, \\
& Z_{21}=-\left.\frac{E_{\phi}}{H_{\theta}}\right|_{H_{\phi}=0}=\frac{b_{21} b_{33}-b_{23} b_{31}}{b_{23} b_{41}-b_{21} b_{43}}, \\
& Z_{12}=\left.\frac{E_{\theta}}{H_{\phi}}\right|_{H_{\theta}=0}=\frac{b_{11} b_{43}-b_{13} b_{41}}{b_{21} b_{43}-b_{23} b_{41}}, \\
& Z_{22}=-\left.\frac{E_{\phi}}{H_{\phi}}\right|_{H_{\theta}=0}=\frac{b_{31} b_{43}-b_{33} b_{41}}{b_{23} b_{41}-b_{21} b_{43}},
\end{aligned}
$$

where $b_{i j}$ designates an element of the $b_{n}$ matrix in (61). These impedances are related to ionospheric reflection coefficients, which lead to a rather involved modal equation for determining the propagation characteristics of the coupled TE and TM modes in the earth-to-ionosphere waveguide [Wait, 1963a]. For vertical dipole excitation, TE modes are not excited and also the coupling between TE and TM modes has been shown to be negligible for a single layer ionosphere [Schumann, 1955; Wait, 1962, p. 269]. This approximation is assumed to apply also to a stratified ionosphere, and the propagation characteristics are determined from the impedance $Z_{12}$ in the same way as for an isotropic ionosphere, although the anisotropic structure of the ionosphere is considered in establishing $Z_{12}$.

The surface impedance $Z_{12}$ is computed initially by setting $S=1$ in $\nu(\nu+1)=\left(k_{0} a S\right)^{2}$ and $Z_{12}$ is used in the appropriate modal equations of ELF or VLF waves for obtaining a first estimate of $S=S_{1}$. Using $S_{1}$, the computation of $Z_{12}$ gives $S_{2}$, and the process is repeated until the sequence of $S_{n}$ converges [Galejs, 1964b].

\section{Discussion}

\subsection{Extremely Low Frequency}

For frequencies in the ELF range Roots $S$ of the modal equation are shown in figures 2 and 3 for the ionospheric model of electronic conductivity of figures 1 and 2 of Galejs [1964b]. In the isotropic ionosphere model $\sigma_{0}$ remains unaltered; $\sigma_{1}=\sigma_{0}$ and $\sigma_{2}$ approaches 0 . The anisotropy of the ionosphere increases $\operatorname{Re} S(\operatorname{ReS}=c / v, c=$ velocity of light, $v=$ phase velocity) and also $\operatorname{Im} S$, that is proportional to the attenuation constant. These increases are most pronounced for the nighttime ionosphere model in the lower frequency range shown in figure 3 . The corresponding attenuation constants are plotted in figure 4. 


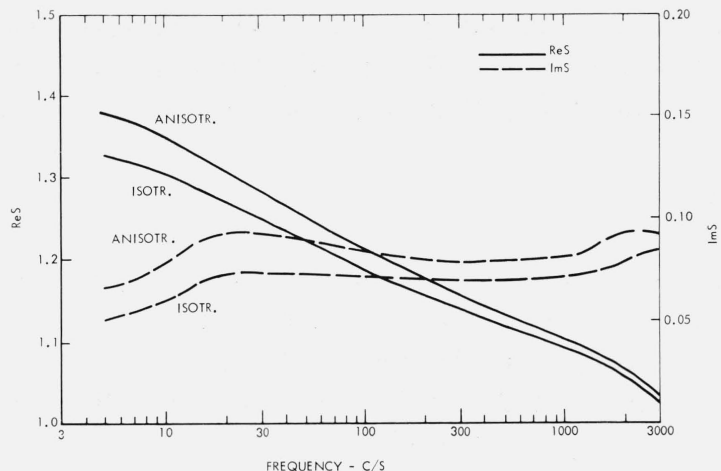

FiguRE 2. Roots of the modal equation, electronic daytime conductivity for $\mathrm{F}=0$.

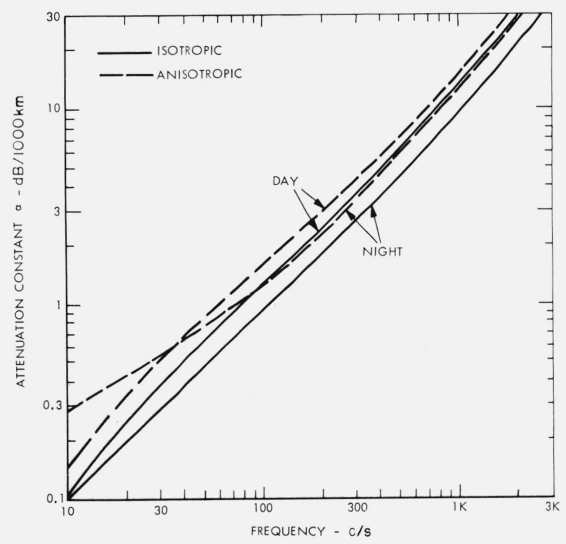

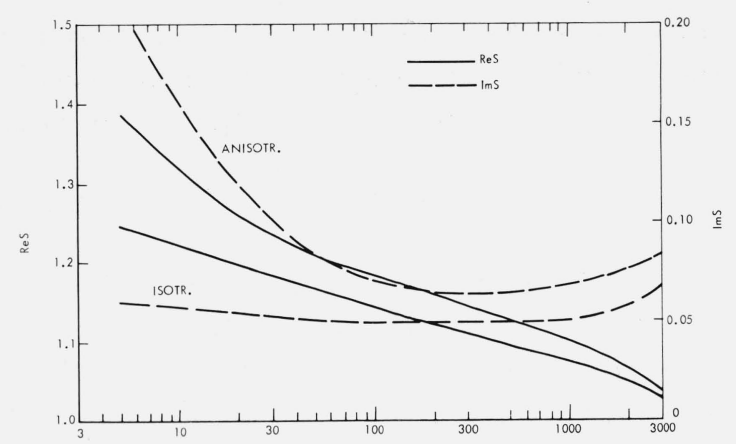

FIGURE 3. Roots of the modal equation, electronic nighttime conductivity for $\mathrm{F}=0$.

FIGURE 4. Attenuation constants, electronic conductivity for $\mathrm{F}=0$.

The resonant frequencies of the earth's ionosphere cavity and its $Q$-factors can be determined from the computed values of $\operatorname{Re} S$ and $\operatorname{Im} S$ [Galejs, 1964a, 1964d]. These calculations are made also for ionosphere models of electronic plus ionic conductivities at $f=0$ and $f=20 \mathrm{c} / \mathrm{s}$ (figs. 1 to 4 of Galejs, 1964b). The calculated resonance frequencies $f_{n}$ are listed in table 1 . The isotropic day or night models exhibit too high resonance frequencies, but the average for the anisotropic day or night models gives nearly correct results although the first resonance occurs near $7.7 \mathrm{c} / \mathrm{s}$. At night the various anisotropic models give different results, while their results are nearly the same for daytime or for the isotropic models. The calculated $Q$-factors are listed in table 2 . At . daytime the $Q$-factors are lowered slightly due to anisotropy. The losses are principally due to absorption in the lower $D$ region, where the collision frequencies are high and the magnetic field has only small effects. At nighttime the anisotropy reduces the $Q$-values drastically and the ions have a considerable effect. For the isotropic models the energy remains below the ionosphere, the losses in the lower ionosphere are small, and the $Q$-values are high. For the anisotropic models some energy penetrates the ionosphere and escapes from the earth-ionosphere cavity. Hence the $Q$ figure is lowered. The present calculations assume the radial magnetic field of the polar regions to be extended over the whole surface of the globe and thus the anisotropy effects of this model should be larger than expected in nature. 
TABLE I

RESONANCE FREQUENCIES OF THE SPHERICAL SHELL BETWEEN THE EARTH AND IONOSPHERE IN THE PRESENCE OF A RADIAL MAGNETIC FIELD

\begin{tabular}{|c|c|c|c|c|c|c|}
\hline & IONOSPHERE MODEL & & 1 & 2 & 3 & 4 \\
\hline \multirow{7}{*}{ 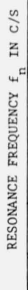 } & DAY, ANISOTROPIC & & 7.8 & 13.8 & 19.8 & 26 \\
\hline & DAY, ISOTROPIC & & 8.1 & 14.2 & 20.4 & 26.6 \\
\hline & \multirow[t]{3}{*}{ NIGHT, ANISOTROPIC } & \multirow{2}{*}{$\begin{array}{l}\text { EL.+ IONS F }=20 \\
E L++ \text { IONS F }=0\end{array}$} & 7.6 & 15.2 & 23.2 & 29.5 \\
\hline & & & 7.7 & 13.8 & 20.1 & 26.9 \\
\hline & & EL. ONLY $\quad F=0$ & 7.8 & 14.1 & 20.7 & 27.8 \\
\hline & \multicolumn{2}{|l|}{ NIGHT, ISOTROPIC } & 8.6 & 15.3 & 21.3 & 27.4 \\
\hline & \multicolumn{2}{|l|}{ MEASUREMENTS } & 8 & 14.1 & 20.3 & 26.4 \\
\hline
\end{tabular}

TABLE II

Q-FACTORS OF EARTH-IONOSPHERE CAVITY RESONANCES IN THE PRESENCE OF A RADIAL MAGNETIC

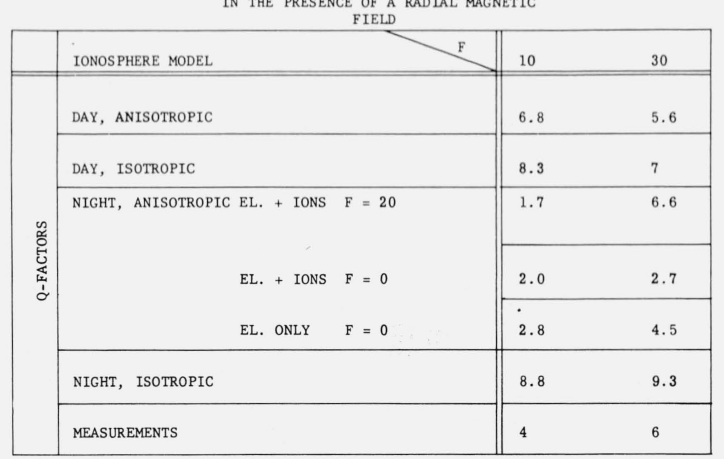

\subsection{Very Low Frequency}

For VLF waves the calculations are made first for a homogeneous sharply bounded ionosphere model in order to obtain a comparison with past results that are based on the quasi-longitudinal approximation [Wait, 1962]. The data shown in figure 5 are calculated for the same ionosphere parameters as in figure 12, page 276, of Wait [1962]. The tensor components of ionospheric conductivity (3) are related to the parameter

$$
\omega_{r}=\frac{\omega_{0}^{2}}{\nu}\left[1+\left(\frac{\omega_{H}}{\nu}\right)^{2}\right]^{-1 / 2}
$$

as

$$
\begin{aligned}
& \sigma_{0}=\epsilon_{0} \omega_{r} \sqrt{1+\left(\omega_{H} / \nu\right)^{2}} \\
& \sigma_{1}=\sigma_{0} /\left[1+\left(\omega_{H} / \nu\right)^{2}\right] \\
& \sigma_{2}=\sigma_{1} \omega_{H} / \nu
\end{aligned}
$$

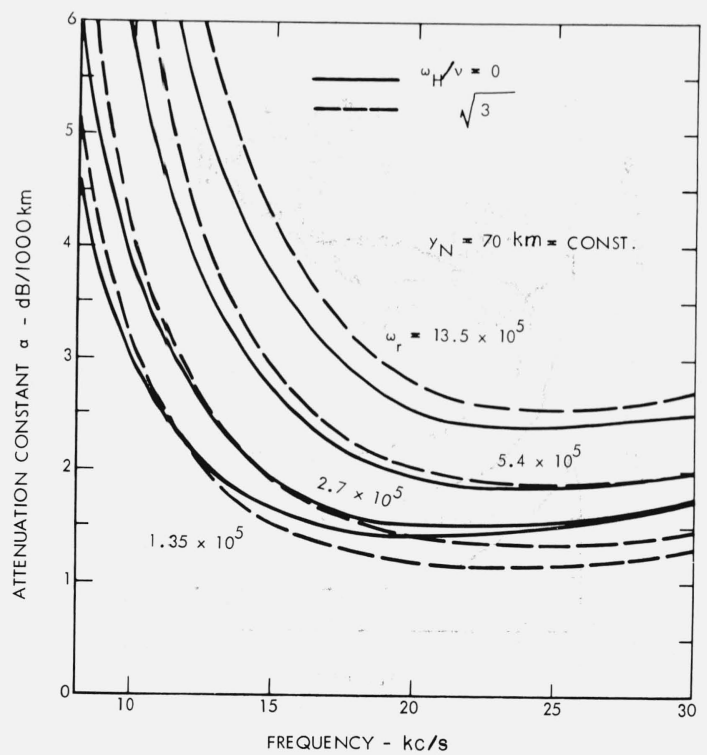

Figure 5. Attenuation rates, sharply bounded homogeneous ionosphere, $\omega_{\mathrm{r}}=$ constant .

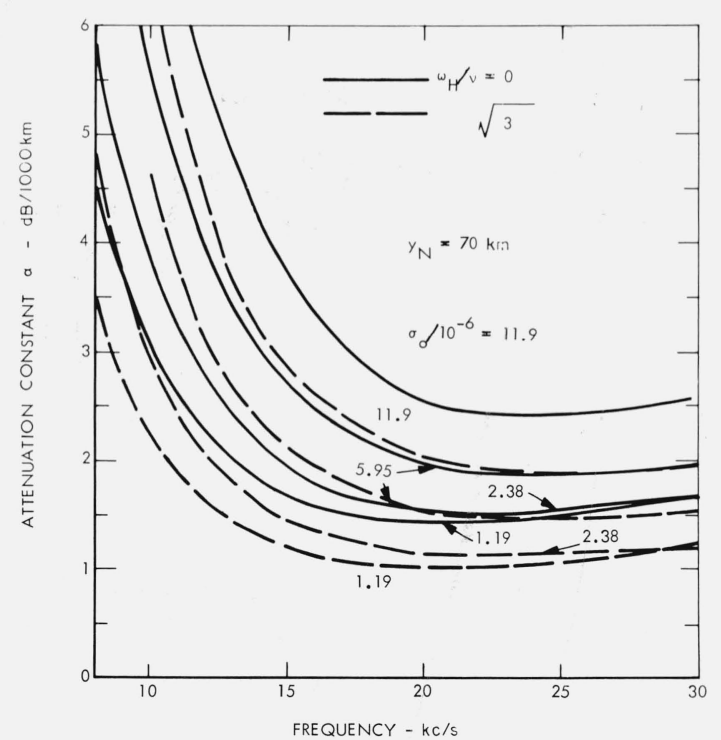

Figure 6. Attenuation rates, sharply bounded homogeneous ionosphere, $\sigma_{0}=$ constant . 
where $\omega_{0}$ is a plasma frequency, $\omega_{H}$ gyrofrequency, and $\nu$ is collision frequency. There is a good argument with the results of Wait for frequencies above $15 \mathrm{kc} / \mathrm{s}$, but the attenuation rates of figure 5 are somewhat higher for frequencies near $10 \mathrm{kc} / \mathrm{s}$. This may be due in part to the differences between the modal equation used by Wait [1962, eq 19, p. 275] and the equations (45) to (50) of Galejs [1964b] which are used for the calculations shown in figure 5. (The differences of attenuation rates that are computed using such modal equations can be seen for a different ionospheric model from figure 14 of Galejs [1964b].)

The attenuation rates can be computed also for $\sigma_{0}$ constant in the isotropic and anisotropic models, as indicated in figure 6 . The attenuation rates are decreased due to the anisotropy in figure 6.

Higher ratios of $\left(\omega_{H} / \nu\right)$ may be appropriate for the nighttime ionosphere when VLF waves may reach higher ionosphere regions where collision frequencies are lower. As an example, calculations are made for $\omega_{H} / \nu=10$ and for the lower ionosphere boundary at $y_{N}=90 \mathrm{~km}$, as shown in figure 7. There is a significant decrease of the attenuation rates due to the magnetic field, particularly for lower ionospheric conductivity.

Propagation parameters are also calculated for the profiles of electronic conductivity for $f=0$ shown in figures 1 and 2 of Galejs [1964b]. At daytime there is hardly a change due to the radial magnetic field, but the nighttime attenuation rates are significantly decreased. This is in line with the data shown in figure 7 for a sharply bounded homogeneous ionosphere model. There are uncertainties associated with nighttime models, and the numerical differences shown in figure 8 may be higher than observed experimentally.

The attenuation rates are also calculated for a daytime ionosphere model which considers electronic and ionic conductivities at $f=15 \mathrm{kc} / \mathrm{s}$ [fig. 5 of Galejs, 1964b]. Selection of two different boundary heights $y_{N}$ of the ionosphere model (ionosphere conductivity is assumed to be zero for $y<y_{N}$ ) causes only minor changes between calculated attenuation rates, as is seen from figure 9. The ionospheric anisotropy decreases $\alpha$ for $y_{N}=60 \mathrm{~km}$, but it has a negligible effect for $y_{N}=50$. The effects of anisotropy are comparable to the uncertainty of the propagation parameters associated with the selection of two different boundary heights $y_{N}$.

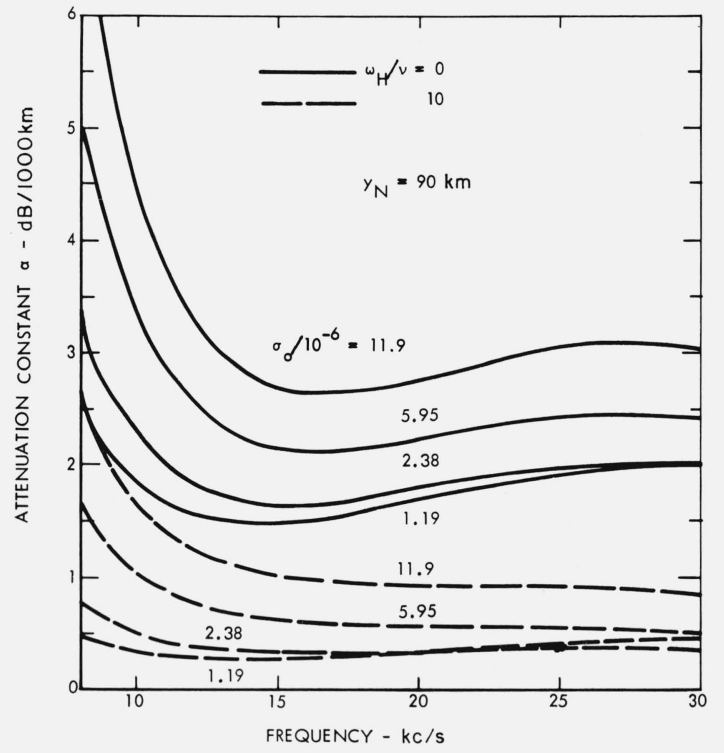

FIG URE 7. Attenuation rates, sharply bounded homogeneous ionosphere, $\sigma_{0}=$ constant.

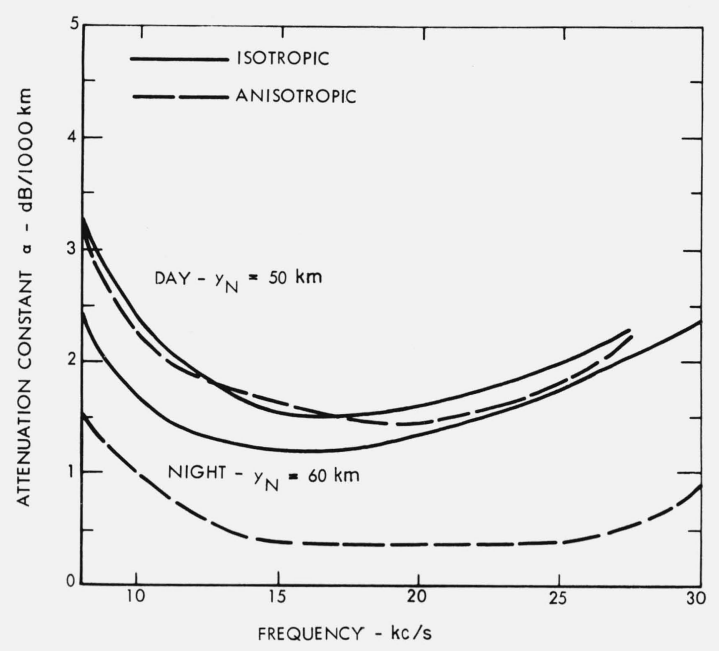

FIGURE 8. Attenuation rates electronic conductivity for $\mathrm{F}=0$. 
Figure 9. Attenuation rates, electronic plus ionic daytime conductivity for $\mathrm{F}=15 \mathrm{kc} / \mathrm{s}$.

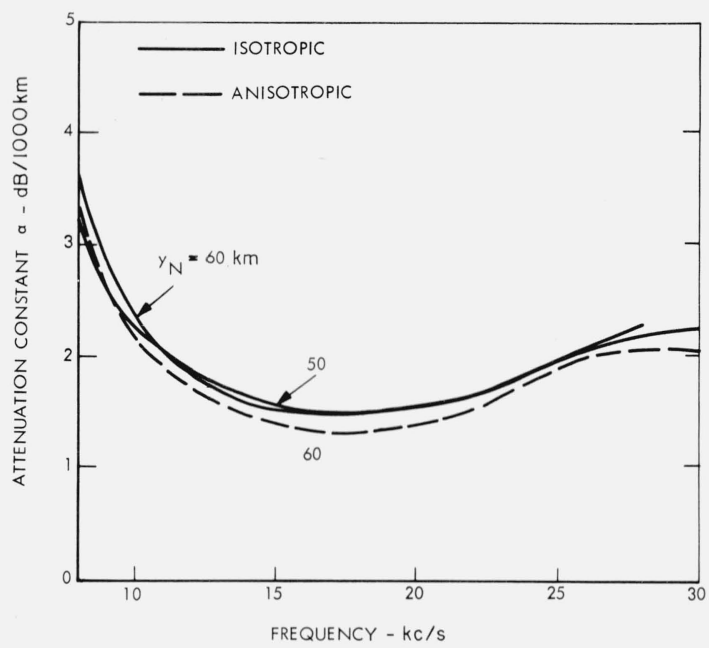

Figures 5 to 9 have shown only the attenuation rates which are proportional to $\operatorname{Im} S$. The changes of $\operatorname{Re} S$ due to the radial magnetic field are negligible and the $\operatorname{Re} S$ figures for an isotropic ionosphere [Galejs, 1964c] apply also to the case of radial magnetic field.

I express appreciation to S. R. Cassarino and E. M. Larsen for computer programming. This work was supported by the Office of Naval Research under contract Nonr 3185(00).

\section{Appendix 1. Matrix $\left[d_{n}\right]$}

After computing the inverse matrix $\left[a_{n}^{-1}\left(r_{n-1}\right)\right]$, the elements of the matrix

$$
\left[d_{n}\right]=\left[a_{n}\left(r_{n}\right)\right] \cdot\left[a_{n}^{-1}\left(r_{n-1}\right)\right]
$$

are computed as

$$
\begin{aligned}
& d_{11}=\frac{1}{1+H G} \frac{r_{n-1}}{r_{n}}\left[\cos k_{+} h+H G \cos k_{-} h\right] \\
& d_{12}=-\frac{i}{1+E F} \frac{r_{n-1}}{r_{n}}\left[\frac{x_{n}}{y_{n}} \sin k_{+} h+\frac{w_{n} G F}{z_{n}} \sin k_{-} h\right] \\
& d_{13}=\frac{G}{1+H G} \frac{r_{n-1}}{r_{n}}\left[-\cos k_{+} h+\cos k_{-} h\right] \\
& d_{14}=\frac{i}{1+E F} \frac{r_{n-1}}{r_{n}}\left[-\frac{x_{n} E}{y_{n}} \sin k_{+} h+\frac{w_{n} G}{z_{n}} \sin k_{-} h\right] \\
& d_{21}=-\frac{i}{1+H G} \frac{r_{n-1}}{r_{n}}\left[\frac{y_{n}}{x_{n}} \sin k_{+} h+\frac{z_{n} E H}{w_{n}} \sin k_{-} h\right] \\
& d_{22}=\frac{1}{1+E F} \frac{r_{n-1}}{r_{n}}\left[\cos k_{+} h+E F \cos k_{-} h\right] \\
& d_{23}=\frac{i}{1+H G} \frac{r_{n-1}}{r_{n}}\left[\frac{y_{n} G}{x_{n}} \sin k_{+} h-\frac{z_{n} E}{w_{n}} \sin k_{-} h\right]
\end{aligned}
$$




$$
\begin{aligned}
& d_{24}=\frac{E}{1+E F} \frac{r_{n-1}}{r_{n}}\left[\cos k_{+} h-\cos k_{-} h\right] \\
& d_{31}=\frac{H}{1+H G} \frac{r_{n-1}}{r_{n}}\left[-\cos k_{+} h+\cos k_{-} h\right] \\
& d_{32}=\frac{i}{1+E F} \frac{r_{n-1}}{r_{n}}\left[\frac{x_{n} H}{y_{n}} \sin k_{+} h-\frac{w_{n} F}{z_{n}} \sin k_{-} h\right] \\
& d_{33}=\frac{1}{1+H G} \frac{r_{n-1}}{r_{n}}\left[\cos k_{-} h+H G \cos k_{+} h\right] \\
& d_{34}=\frac{i}{1+E F} \frac{r_{n-1}}{r_{n}}\left[\frac{x_{n} H E}{y_{n}} \sin k_{+} h+\frac{w_{n}}{z_{n}} \sin k_{-} h\right] \\
& d_{41}=\frac{i}{1+H G} \frac{r_{n-1}}{r_{n}}\left[-\frac{y_{n} F}{x_{n}} \sin k_{+} h+\frac{z_{n} H}{w_{n}} \sin k_{-} h\right] \\
& d_{42}=\frac{F}{1+E F} \frac{r_{n-1}}{r_{n}}\left[\cos k_{+} h-\cos k_{-} h\right] \\
& d_{43}=\frac{\mathrm{i}}{1+H G} \frac{r_{n-1}}{r_{n}}\left[\frac{y_{n} F G}{x_{n}} \sin k_{+} h+\frac{z_{n}}{w_{n}} \sin k_{-} h\right] \\
& d_{44}=\frac{1}{1+E F} \frac{r_{n-1}}{r_{n}}\left[\cos k_{-} h+E F \cos k_{+} h\right]
\end{aligned}
$$

where the symbols are defined as in (64) to (72) and where $h=r_{n-1}-r_{n}$. However, these expressions are valid only if $N^{\prime}=N^{\prime \prime}$ in (69) to (72).

When neglecting ionospheric displacement currents $q_{j}$ 's are small and

$$
F=H=-G=-E=-i
$$

and the matrix elements simplify to

$$
\begin{aligned}
& d_{11}=d_{22}=d_{33}=d_{44}=\frac{r_{n-1}}{2 r_{n}}\left(\cos k_{+} h+\cos k_{-} h\right) \\
& d_{12}=-d_{34}=-\frac{i \omega \mu_{0}}{2} \frac{r_{n-1}}{r_{n}}\left[\frac{\sin k_{+} h}{k_{+}}+\frac{\sin k_{-} h}{k_{-}}\right] \\
& d_{13}=-d_{31}=-d_{24}=d_{42}=\frac{i}{2} \frac{r_{n-1}}{r_{n}}\left[-\cos k_{+} h+\cos k_{-} h\right] \\
& d_{14}=d_{32}=\frac{\omega \mu_{0}}{2} \frac{r_{n-1}}{r_{n}}\left[\frac{\sin k_{+} h}{k_{+}}-\frac{\sin k_{-} h}{k_{-}}\right] \\
& d_{21}=-d_{43}=-\frac{i}{2 \omega \mu_{0}} \frac{r_{n-1}}{r_{n}}\left[k_{+} \sin k_{+} h+k_{-} \sin k_{-} h\right] \\
& d_{23}=d_{41}=-\frac{1}{2 \omega \mu_{0}} \frac{r_{n-1}}{r_{n}}\left[k_{+} \sin k_{+} h-k_{-} \sin k_{-} h\right] .
\end{aligned}
$$

The coefficients (102) to (107) are in agreement with results of Thompson [1963], which were originally obtained starting out with the field representation (38) to (43).

For uncoupled TE and TM modes, $E, H, F$, and $G$ approach zero and the matrix elements (85) to (100) become 


$$
\begin{aligned}
& d_{11}=d_{22}=\frac{r_{n-1}}{r_{n}} \cos k_{+} h \\
& d_{12}=-\frac{i \omega \mu_{0}\left(1-q_{3}\right)}{k_{+}} \frac{r_{n-1}}{r_{n}} \sin k_{+} h \\
& d_{13}=d_{14}=d_{23}=d_{24}=d_{31}=d_{32}=d_{41}=d_{42}=0 \\
& d_{21}=\frac{k_{+}}{i \omega \mu_{0}\left(1-q_{3}\right)} \frac{r_{n-1}}{r_{n}} \sin k_{+} h \\
& d_{33}=d_{44}=\frac{r_{n-1}}{r_{n}} \cos k_{-} h \\
& d_{34}=\frac{i \omega \mu_{0}}{k_{-}} \frac{r_{n-1}}{r_{n}} \sin k_{-} h \\
& d_{43}=-\frac{k_{-}}{i \omega \mu_{0}} \frac{r_{n-1}}{r_{n}} \sin k_{-} h .
\end{aligned}
$$

After expanding the sine and cosine functions and substituting the values of $k_{+}$and $k_{-}$from (28) and (30), the coefficients (108) to (114) are in agreement with the free space results of Thompson [1963], which were originally obtained from a Taylor series expansion of Hankel functions representing the fields between earth and ionosphere. The matrix elements (108) to (114) were derived using the planar approximation (14). These expressions remain valid in the ELF range in the spherical shell between the ground and ionosphere. For VLF, these expressions should be used only in thin shells like in the vicinity of the ionospheric boundary.

\section{Appendix 2. Multipliers $e_{i j}$}

After carrying out the matrix multiplications

$$
\begin{aligned}
{\left[b_{1}\right] } & =\left[d_{1}\right] \cdot\left[a_{n}\left(r_{0}\right)\right]=\left[d_{1}\right] \cdot\left[b_{0}\right] \\
{\left[b_{2}\right] } & =\left[d_{2}\right] \cdot\left[b_{1}\right] \\
& \vdots \\
{\left[b_{n}\right] } & =\left[d_{n}\right] \cdot\left[b_{n-1}\right]
\end{aligned}
$$

it is found that the ratio between an element of the matrix $\left[b_{n}\right]$ and the corresponding element of the matrix $\left[b_{n-1}\right]$ is given by

$$
e_{i j}=\left.b_{i j}\right|_{n} /\left.b_{i j}\right|_{n-1}
$$

and it does not depend on other elements of the $\left[b_{n-1}\right]$ matrix if $E, F, G$, and $H$ of two adjacent layers are the same. The multipliers $e_{i j}$ are obtained as follows:

$$
\begin{aligned}
& e_{11}=e_{31}=\left(\cos k_{+} h-i \frac{x_{n}}{y_{n}} \frac{y_{n-1}}{x_{n-1}} \sin k_{+} h\right) \frac{r_{n-1}}{r_{n}} \\
& e_{12}=e_{32}=\left(\cos k_{+} h+i \frac{x_{n}}{y_{n}} \frac{y_{n-1}}{x_{n-1}} \sin k_{+} h\right) \frac{r_{n-1}}{r_{n}} \\
& e_{13}=e_{33}=\left(\cos k_{-} h-i \frac{w_{n}}{z_{n}} \frac{z_{n-1}}{w_{n-1}} \sin k_{-} h\right) \frac{r_{n-1}}{r_{n}}
\end{aligned}
$$




$$
\begin{aligned}
& e_{14}=e_{34}=\left(\cos k_{-} h+i \frac{w_{n}}{z_{n}} \frac{z_{n-1}}{w_{n-1}} \sin k_{-} h\right) \frac{r_{n-1}}{r_{n}} \\
& e_{21}=e_{41}=\left(\cos k_{+} h-i \frac{y_{n}}{x_{n}} \frac{x_{n-1}}{y_{n-1}} \sin k_{+} h\right) \frac{r_{n-1}}{r_{n}} \\
& e_{22}=e_{42}=\left(\cos k_{+} h+i \frac{y_{n}}{x_{n}} \frac{x_{n-1}}{y_{n-1}} \sin k_{+} h\right) \frac{r_{n-1}}{r_{n}} \\
& e_{23}=e_{43}=\left(\cos k_{-} h-i \frac{z_{n}}{w_{n}} \frac{w_{n-1}}{z_{n-1}} \sin k_{-} h\right) \frac{r_{n-1}}{r_{n}} \\
& e_{24}=e_{44}=\left(\cos k_{-} h+i \frac{z_{n}}{w_{n}} \frac{w_{n-1}}{z_{n-1}} \sin k_{-} h\right) \frac{r_{n-1}}{r_{n}} .
\end{aligned}
$$

\section{References}

Galejs, J. (1964a), Terrestrial extremely-low-frequency propagation, Proc. NATO Advanced Study Institute on Natural Electromagnetic Phenomena Below 30 kc/s, Bad Homburg, W. Germany, July-August 1963, pp. 205-258 (Plenum Press, New York, N.Y.).

Galejs, J. (June 1964b), ELF and VLF waves below an inhomogeneous anisotropic ionosphere, Radio Sci. J. Res. NBS 68D, No. 6, 693-707.

Galejs, J. (Sept. 1, 1964c), Propagation of VLF waves below a curved and stratified anisotropic ionosphere, J. Geophys. Res. 69, No. 17, 3639-3650.

Galejs, J. (1964d), Schumann resonances, review paper for the ULF Symposium, Boulder, Colo., August 17 to 20.

Galejs, J., and R. V. Row (Jan. 1964), Propagation of ELF waves below an inhomogeneous anisotropic ionosphere, IEEE Trans. Ant. Prop. AP-12, No. 74-83; also Radio Sci. J. Res. NBS 68D, No. 1, 103-104.

Schumann, W. O. (1955), Der Einfluss des Erdmagnetfeldes auf die Ausbreitung elektrischer Laengstwellen, Zeitschrift fur angewandte Physik 7, No. 6, 284-290.

Thompson, W. B. (Feb. 1963), A layered model approach to the earth-ionosphere cavity resonance problem, Ph. D. thesis, Department of Geology and Geophysics, Massachusetts Institute of Technology.

Volland, H. (1962), The propagation of plane electromagnetic waves in a horizontally stratified ionosphere, J. Atmospheric Terrest. Phys. 24, 853-857.

Volland, H. (1964), Zur Theorie der Ausbreitung langer elektromagnetischer Wellen Teil II: Gekruemmter anisotroper Wellenleiter, Heinrich-Hertz-Institut-für Schwingungsforschung Berlin-Charlottenburg. Archiv der Elecktrischen Uebertragung 18, No. 3, 181-188.

Wait, J. R. (1962), Electromagnetic waves in stratified media (Macmillan Co., New York, N.Y.).

Wait, J. R. (1963a), The mode theory of VLF radio propagation for a spherical earth and a concentric anisotropic ionosphere, Can. J. Phys. 41, 299-315.

Wait, J. R. (May-June 1963b), Concerning solutions of the VLF mode problem for an anisotropic curved ionosphere, J. Res. NBS 67D (Radio Prop.), No. 3, 297-302.

\section{Additional Reference}

Price, G. H. (April 1964), Propagation of electromagnetic waves through a continuously varying stratified anisotropic medium, Radio Sci. J. Res. NBS 68D, No. 4, 407-418.

(Paper 69D5-506) 